ИЗВЕСТИЯ АКАДЕМИИ НАУК ЭСТОНСКОИ ССР. ФИЗИКА * МАТЕМАТИКА

PROCEEDINGS OF THE ACADEMY OF SCIENCES OF THE ESTONIAN SSR.

PHYSICS * MATHEMATICS

$1987,36,3$

удК [535.217:535.34] :539.219.1

Я. КНКАС, К. ХАЛЛЕР

\title{
НЕРЕЗОНАНСНОЕ ЛАЗЕРНОЕ ВОЗДЕИСТВИЕ НА ПРИМЕСНОЕ СТЕКЛО ПРИ 6 К: ГИСТЕРЕЗИСНЫЕ И ПОРОГОВЫЕ ЯВЛЕНИЯ В КИНЕТИКЕ ФОТОВЫЖИГАНИЯ
}

J. KIKAS, K. HALLER. LISANDIKLAASI MITTERESONANTNE LASERMOJUTUS TEMPERATUURIL $6 \mathrm{~K}$ : HOSTEREES JA LAVINAHTED VALGUSSALKAMISE KINEETIKAS

J. KIKAS, K. HALLER. NONRESONANT LASER ACTION ON AN IMPURITY GLASS AT 6 K: HYSTERESIS AND THRESHOLD EFFECTS IN SPECTRAL HOLEBURNING

\section{(Представил К. К. Ребане)}

1. Благодаря методам селективной лазерной спектроскопии, особенно методу фотовыжигания спектральных провалов в примесных спектрах $\left[{ }^{1,2}\right]$, накоплена богатая экспериментальная информация о (равновесных) процессах в низкотемпературных аморфных средах $\left[{ }^{3}\right]$. Вместе с тем вопрос о влиянии лазерного излучения на аморфные примесные системы, кроме феноменологически относительно тривиального эффекта селективного выжигания $[1,4,5]$, изучен весьма слабо. Отчасти трудности связаны с тем, что монохроматическое лазерное облучение выполняет двоякую роль - как зонд и источник исследуемых процессов. В экспериментах по определению равновесных характеристик (однородных ширин линий, например) последнее влияние обычно пытаются свести к минимуму (ограничения на интенсивность, время и дозу облучения). Имеющиеся сведения о нетривиальном воздействии лазерного излучения на примесные стекла (предположение о светоиндуцированной спектральной диффузии [ $\left.{ }^{6}\right]$, световое стирание спектральных провалов, не коррелирующее с эффективностью примесного поглощения $\left[{ }^{7}\right]$ ) весьма скудны и не имеют когерентной интерпретации.

В настоящем сообщении обнаружено и исследовано влияние нерезонансного лазерного излучения на кинетику спектрально селективного фотовыжигания примеси тетрацена в аморфной матрице этанола.

2. Насыщенный раствор тетрацена в этаноле (концентрация $2 \cdot 10^{-5}$ моль/л), находящийся в плоскопараллельной кварцевой кювете толщиной $0,2 \mathrm{mм}$, охлаждался со скоростью $\geqslant 1 \mathrm{~K} \mathrm{c}^{-1}$ до температуры $6 \mathrm{~K}$ в парах гелия. Образовавшийся при этом оптически качественный образец стеклообразного твердого раствора поддерживался в гелиевом криостате типа УТРЕКС при температуре $6,0 \pm 0,1$ К. Облучение его осуществлялось двумя линиями $\left(\lambda_{1}=476,5\right.$ нм и $\lambda_{2}=514,5$ нм) аргоновых лазеров (ILA-120 и INNOVA 100-20 соответственно), мощность излучения которых стабилизировалась с точностью $\pm 2 \%$. Диафрагмированные лазерные лучи проходили отверстие диаметром 1 мм в поставленной непосредственно перед кюветой маске и выводились из криостата. Возбуждение флуоресценции тетрацена осуществлялось линией $\lambda_{1}$, попавшей в неоднородную $0-0$-полосу $S_{1} \leftarrow S_{0}$-поглощения примеси (максимум при 475 нм, полуширина $\approx 250 \mathrm{~cm}^{-1}$ ). Селективно возбужденная флуоресценция регистрировалась на максимуме выбронной ли- 
нии $v_{00}-310 \mathrm{~cm}^{-1}\left[{ }^{8}\right]$ через двойной монохроматор «Spex 1402» (голографические решетки, ширина щелей $2,5 \mathrm{~cm}^{-1}$ ) в режиме счета фотонов с помощью программируемого многоканального анализатора LP 4900.

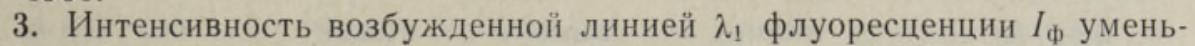
шается со временем из-за процесса фотовыжигания $\left[{ }^{8,9}\right]$ (см. на рисунке кривые $1,1^{\prime}$ ). С включением света $\lambda_{2}$ (кривые $3,3^{\prime}$ ) наблюдается резкий скачок интенсивности флуоресценции $I_{\text {ф }}$ на $\Delta_{1}$. Время скачка меньше 0,2 с (максимально использованное временное разрешение). Поведение сигнала флуоресценции при выключении света $\lambda_{2}$ существенным образом зависит от интенсивности $I_{2}$ (сравни кривые $1,1^{\prime}$ на рисунке, $a)$. При $I_{2} \leqslant 2$ Вт·см ${ }^{-2}$ наблгдается непрерывный спад интенсивности $I_{\phi}$ с характерным временем $15-35$ с (см. ниже пункт 3.8). При $I_{2} \geqslant 2 \mathrm{BT} \cdot \mathrm{cm}^{-2}$ спаду предшествует резкий скачок интенсивности $I_{\phi}$ на $\Delta_{2}$ - зависимость $I_{\Phi}\left(I_{2}\right)$ проявляет гистерезисный характер. Появление скачка $\Delta_{2}$ имеет ярко выражәнғый пороговой характер (на вставке рисунка, б приведена зависимость амплитуды скачка от интснсивности света $I_{2}$ ). С появлением скачка $\Delta_{2}$ коррелирует также насыщәние амплитуды скачка $\Delta_{1}$ при росте $I_{2}$. Зависимость $\Delta_{1}\left(I_{2}\right)$ (кривая $I$ на рисунке, б в промежутке времени от 660 до 730 с) снята линсїным по времени сканированием $I_{2}$ (кривая 3 на рисунке, б):

Дополнительно нами установлено следующее:

3.1. Облучение только светом с длиной волны $\lambda_{2}$ не вызывает свечения образца и не дает добавки к темновому фону.

3.2. Скачков интенсивности свечсния образца не наблюдается после растройки длины волны регнстрации на $\sim 15 \mathrm{~cm}^{-1}$ в коротковолновую сторону от вибронной линии флуоресценции.

3.3. Не обнаружено длительного послесвечения образца (свечение затухает за время $<200$ мс после выключения света $\left.\lambda_{1}\right)$.

Временные зависимости интенсивности вибронной $\left(v_{00}=\right.$ $=310 \mathrm{~cm}^{-1}$ ) линии флуоресценции тетрацена в твердом этаноле при бихроматическом лазерном облученин: резонансно в 0 -0-полосе $\left(\lambda_{1}=\right.$ $=476,5$ нм, $\left.I_{1}=20 \mathrm{MBT} \cdot \mathrm{cm}^{-2}\right)$ и вне резонанса $\left(\lambda_{2}=514,5\right.$ нм, $I_{2}=4 \mathrm{BT} \cdot \mathrm{cm}^{-2}(1)$ и $1 \mathrm{BT} \cdot \mathrm{cm}^{-2}$ ( $\left.I^{\prime}\right)$ ) (a). Кривые $1, I^{\prime}$ сдвинуты друг относительно друга в вертикальном направлении. При $t=240$ с величина $I_{\phi}$ составляет $45 \%$ начального значения $(t=0)$. Временной ход интенсивностей лазеров описывают кривые 2, 3 (соответствуют 1 ) и $2,3^{\prime}$ (соответствуют $\left.I^{\prime}\right)$. Начало отсчета времени здесь и в части б- момент включения света $\lambda_{1}$.

Те же временные зависимости при линейном сканировании интенсивности света $I_{2}$ от 1 до $4 \mathrm{BT} \cdot \mathrm{cm}^{-2}$ (б). $\mathrm{Ha}$ вставке приведена зависимость амплитуды второго скачка интенсивности флуоресценции $\Delta_{2}$ от интенсивности света $I_{2}$.
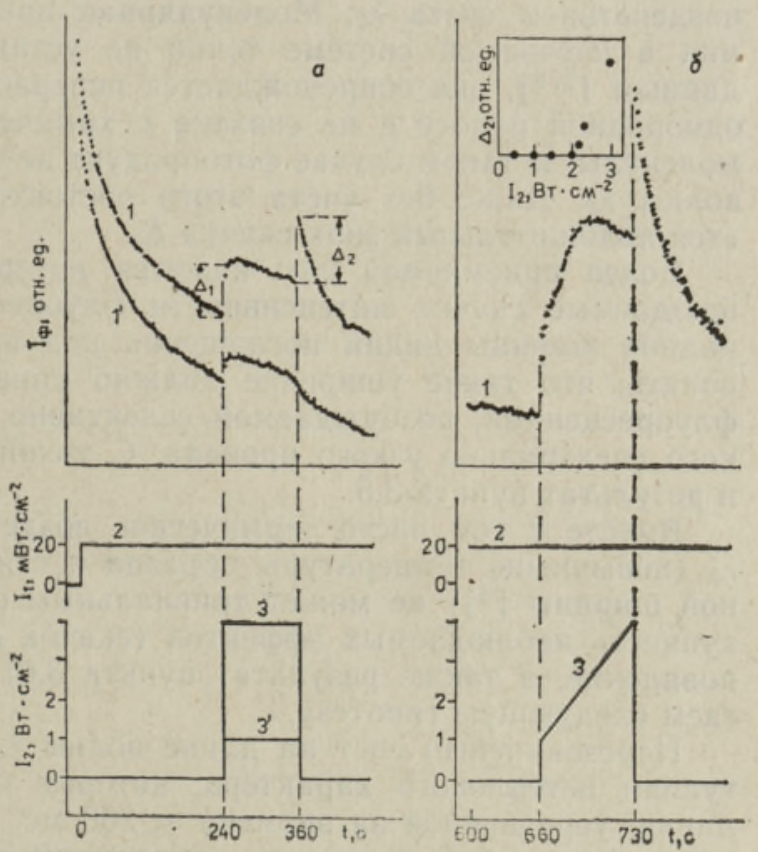
3.4. Скачок интенсивности $\Delta_{1}$ и характерный спад после выключения света $\lambda_{2}$ наблюдается и в случае, когда во время его воздействия свет $\lambda_{1}$ был выключен и включался одновременно с выключением света $\lambda_{2}$.

3.5. Амплитуда скачка $\Delta_{1}$ зависит от общей дозы облучения светом $\lambda_{1}$, обнаруживая быстрый рост в начальной стадии облучения, максимум при времени облучения $360 \pm 20$ с $\left(I_{1}=20 \mathrm{mBT} \cdot \mathrm{cm}^{-2}\right)$ и последующий весьма медленный спад.

3.6. Добавление к раствору примеси, поглощающей излучение $\lambda_{2}$ (родамин 6Ж, концентрация $5 \cdot 10^{-4}$ моль/л, оптическая плотность в $0,2$ мм слое 0,04$)$, не влияет на наблюдаемые эффекты.

3.7. Медленное $\left(\preccurlyeq 0,1 \mathrm{~K} \cdot \mathrm{c}^{-1}\right)$ охлаждение раствора после выдержки в течение двух часов при температуре $150 \mathrm{~K}$, приводящее к образованию сильно потресканного образца, существенно меняет картину. Исчезает скачок $\Delta_{1}$, при $I_{2} \geqslant 2 \mathrm{BT} \cdot \mathrm{cm}^{-2}$ скачок $\Delta_{2}$ остается, но время его релаксации увеличивается.

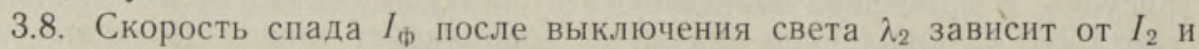
времени его воздействия. При $I_{2}=1,2$ и 4 Вт.см ${ }^{-2}$ (остальные условия см. на рисунке) характерные времена спада $I_{\text {Ф }}$ составляли 35,15 и $50( \pm 5)$ с соответственно.

4. Результаты $3.1-3.3$ доказывают, что наблюдаемые эффекты имеют действительно место в флуоресцентном излучении молекул тетрацена, возбуждаемых селективно через чисто электронную линию.

Существует ряд причин, не позволяющих объяснить наблюдаемые скачки интенсивности флуоресценции перераспределением заселенностей $S_{0}$ и $T_{1}$-состояний под воздействием света $\lambda_{2}=514,5$ нм вследствие $T-T$-поглощения. Согласно $\left[{ }^{10}\right]$, время жизни $T_{1}$-состояния молекулы тетрацена составляет 1 мс, что на 3-4 порядка короче наблюдаемого

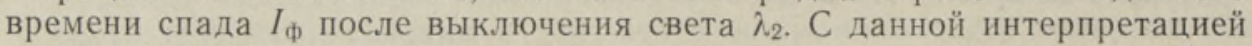
не согласуются также зависимость амплитуды скачка $\Delta$ : от дозы облучения светом $\lambda_{1}=476,5$ нм $(3,5)$ и положительный знак скачка $\Delta_{2}$.

Неудовлетворительным представляется также возможное объяснение эффекта с помощью изменения фотохимического равновесия под воздействием света $\lambda_{2}$. Молекулярная природа процесса фотовыжигания в изучаемой системе точно не установлена, но, по имеющимся данным $\left[{ }^{8,9}\right]$, она сопровождается перераспределением примеси по неоднородной полосе и не связана с химической трансформацией самой молекулы. В таком случае фотопродукт не способен поглощать на длине волны $\lambda_{2}$. Даже без учета этого обстоятельства необъяснимым остается положительный знак скачка $\Delta_{2}$.

Более приемлемой нам кажется интерпретация, связывающая наблюдаемые скачки интенсивности флуоресценцин с изменением однородной ширины линии поглощения под воздействием света $\lambda_{2}$. Легко понять, что такое уширение должно привести к росту интенсивности флуоресценции, возбуждаемой селективно «на дне» достаточно глубокого спектрально узкого провала. С такой интерпретацией согласуется и результат пункта 3.5 .

Вместе с тем чисто термическое воздействие нерезонансного света $\lambda_{2}$ (повышение температуры образца и связанный с ним рост однородной ширины $\left.\left[{ }^{3}\right]\right)$ не может тривиальным образом объяснить всю совокупность наблюдаемых эффектов (скачок $\Delta_{2}$ и пороговый характер его появления, а также результат пункта 3.6). В связи с этим мы выдвигаем следующую гипотезу.

Нерезонансный свет на длине волны $\lambda_{2}$ индуцирует в стекле флуктуации нетеплового характера, которые обусловливают рост ширины линии, усредненной за времена $<200$ мс. Нижний предел для частоты таких флуктуаций по нашим измерениям 5 Гц. С ростом иңтенсивности 
$I_{2}$ растет амплитуда флуктуации, что при $I_{2} \geqslant 2 \mathrm{BT} \cdot \mathrm{cm}^{-2}$ приводит к образованию новой «фазы», стабилизированной воздействием света $\lambda_{2}$. Переход сопровождается поглощением определенного количества тепла. Установление температурного равновесия после такого перехода отражается и в кинетике флуоресценции (ср. ход кривых $1,1^{\prime}$ на рисунке, $a$ после включения света $\lambda_{2}$ ). При выключенй света $\lambda_{2}$ новая «фаза» быстро распадается с выделением тепла. Это обусловливает температурный рост однородной ширины и, как результат, скачок интенсивности флуоресценции $\Delta_{2}$. Выяснение микромеханизма предполагаемого светоиндуцированного «фазового» перехода требует дальнейших исследований.

\section{Л ИТ Е Р А Т Р А}

1. Rebane, L., Gorokhovskii, A., Kikas, J. Appl. Phys. B, 29, № 2, 235-250 (1982).

2. Rebane, K. K., Rebane, L. A. In: Persistant Spectral Hole Burning: Science and Applications (Ed. W. E. Moerner), Springer-Verlag, Heidelberg, 1987 (in press).

3. Rebane, K. K., Gorokhovskii, A. A. J. Lumin., 36, № 4/5, 237-250 (1987).

4. Гороховский А. А., Кикас Я. В. ЖПС, 28, № 5, 832-837 (1978).

5. Jalmukhambetov, A. V., Osad'ko, I. S. Chem. Phys., 77, № 2, 247-255 (1983).

6. Гороховский A. А., Корровитс В. Х., Пальм В. В., Труммал М. А. Письма в ЖЭТФ, 42, № 6, 249-252 (1985).

7. Feary, B. L., Carter, T. P., Small, G. J. Chem. Phys., 101, № 2, 279-286 (1986).

8. Гороховский А. А., Кикас Я. В., Пальм В. В., Ребане Л. А. ФТТ, 23, № 4, 10401047 (1982).

9. Hayes, J. M., Small, G. J. Chem. Phys., 27, № 1, 151-157 (1978).

10. Мак-Глинн C., Адзуми T., Киносита М. Молекулярная спектроскопия триплетного состояния. М., «Мир», 1972.

Ннститут физики

Академии наук Эстонской ССР
Поступила в редакцию

9/III 1987 